\title{
Software
}

\section{Gestores de referencias de última generación: análisis comparativo de RefWorks, EndNote Web y Zotero}

\author{
Por José A. Cordón-García, Helena Martín-Rodero y Julio Alonso-Arévalo
}

\begin{abstract}
Resumen: Una de las herramientas de mayor utilidad para investigadores y bibliotecarios son los gestores de referencias por su capacidad para recopilar, almacenar y dar formato a la información de diferentes productos, fuentes y tipos de documento. En los últimos años ha surgido una nueva generación de programas que incorporan aplicaciones del nuevo contexto tecnológico que han contribuido a reforzar sus capacidades y potencias. Se hace un análisis general de estas herramientas y sus aplicaciones, así como un estudio comparativo de los diversos productos para destacar los puntos fuertes y los aspectos mejorables de cada uno de ellos.

Palabras clave: Gestores de referencias, Referencias bibliográficas, Evaluación, Investigación, Investigadores.

Title: Generation reference management software: comparative analysis of RefWorks, EndNote web and Zotero
\end{abstract}

Abstract: Reference managing tools are one of the most useful devices for researchers and librarians due to their ability to compile, store and format information related to different products, sources and types of records. In recent years a new generation of reference-managing software has appeared. These new tools include applications from the new technological context that have contributed to reinforcing their capacity and potential. An overview of these tools and their applications is offered. We also makes a comparative analysis of the different products with a view to highlighting their strengths as well as the elements that could be improved in each tool.

Keywords: Reference management software, Bibliographic references, Evaluation, Research, Researchers.

Cordón-García, José A.; Martín-Rodero, Helena; Alonso-Arévalo, Julio. "Gestores de referencias de última generación: análisis comparativo de RefWorks, EndNote web y Zotero". El profesional de la información, 2009, julio-agosto, v. 18 , n. 4, pp. 445-454.

DOI: $10.3145 /$ epi.2009.jul.14

\section{Introducción}

LAS OBRAS DE REFERENCIA EN GENERAL y las fuentes de carácter bibliográfico en particular constituyen el ejemplo más claro en el contexto documental de adaptación permanente, siste- mática e inmediata al ecosistema de la información.

Desde que Diderot y d'Alembert "inventaran" un dispositivo informativo tan revolucionario como la época en la que tuvo lugar, las fuentes de información se han ido adaptando a los distintos contextos tecnológicos e informativos que se han sucedido a lo largo del tiempo, con la pretensión última de facilitar la identificación de los recursos bibliográficos existentes, así como su acceso y uso. 


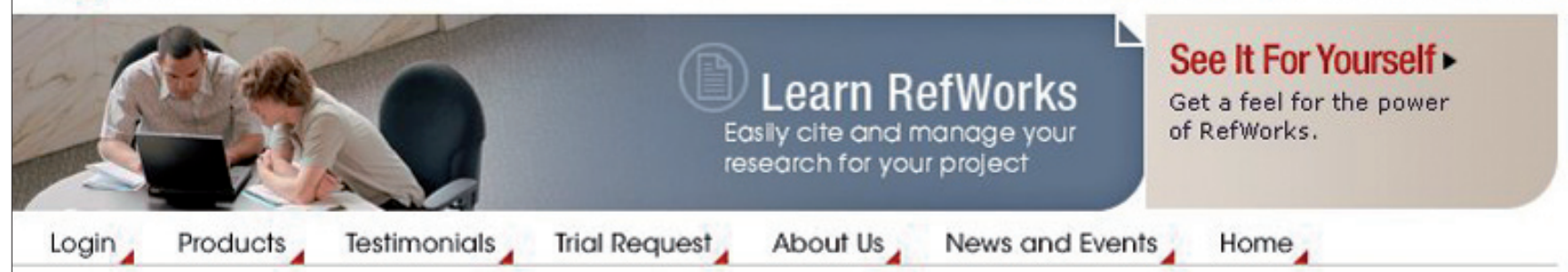

\section{Alrededor del mundo con RefWorks \\ España y Latino-América}

\section{Worldwide \\ Sites}

\begin{abstract}
RefWorks ha creado este sitio en español para apoyar a nuestros usuarios alrededor del mundo con documentación de soporte y formación. Estos sitios proporcionan una diversidad de material desarrollado por RefWorks así como por instituciones subscriptoras que gentilmente han acordado compartir sus recursos. El tipo de material en estos sitios varía e incluye elementos tales como Guías de Inicio Rápido, tutoriales, documentación de ayuda en Español, Gallego, Catalán, etc. y nuestra programación de "webinars". Nuestro sitio le ayuda a aprovechar al máximo su cuenta de RefWorks.
\end{abstract}

Dondequiera se encuentre: iobtenga ayuda de RefWorks fácil e instantáneamente!

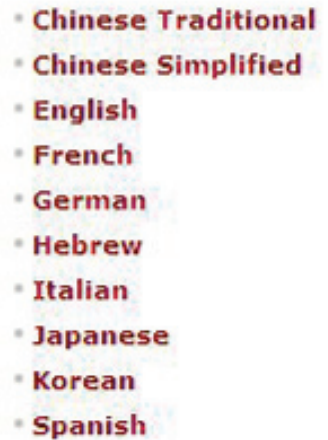

How RefWorks

Can Help You

http://www.refworks.com/es/

En el ámbito de las bibliografías la depuración de los sistemas documentales, la interconexión de fuentes, y el impulso suscitado por la generalización de la Web, ha motivado el crecimiento exponencial de la información disponible para el investigador y el profesional.

De esta manera a los problemas de identificación y búsqueda se ha añadido, cada vez con mayor importancia, el de la organización de la información encontrada. El investigador tiene a su disposición decenas de bases de datos y otras fuentes de información, miles de sitios web, repositorios, blogs, documentos a texto completo, archivos digitalizados de todo tipo, pero se encuentra con la dificultad de la organización de la información que pueda suscitar su interés, de la homogeneización de la misma, y de su optimización desde el punto de vista de la investigación.

Con objeto de resolver los problemas asociados a la gestión hay programas que facilitan estas labores de recopilación, sistematización e integración de recursos e investigación. Son los gestores de referencias bibliográficas que, con ProCite como pionero, vienen implementándose desde hace años.

\section{"El investigador tiene dificultad para organizar la información que le interesa para la investigación"}

Los gestores comparten una serie de funciones básicas:

- Entrada de datos. Puede realizarse de forma manual o automatizada desde el propio gestor de referencias.

- Almacenamiento y recuperación de información. Posibilidad de almacenar de manera estructurada la información (formato RIS ${ }^{1}$ ) y recuperarla fácilmente mediante cualquier punto de acceso.
- Formatos de salida. A partir de los resultados de búsqueda el programa proporciona diversos formatos de salida, como bibliografías, citas bibliográficas a pie de texto, sitios web o bases de datos en internet; y también permite dar forma e insertar citas o bibliografías desde el procesador de textos (Word) en los principales estilos normalizados (ANSI, Vancouver, Chicago, $U M I . .$.$) , en los estilos de publica-$ ción de las instituciones y revistas más conocidas de cada campo del conocimiento (MLA, ACS, ALA, Biochemistry, AIAA Journal) o en el formato documental que nosotros deseemos (rtf, doc, pdf, html).

- Confección de estudios bibliométricos. Los gestores pueden medir el número de ocurrencias de los términos indizados y a partir de ellos elaborar diferentes estudios de carácter cuantitativo.

- Control de autoridades y generación de bibliografías en formato html. 


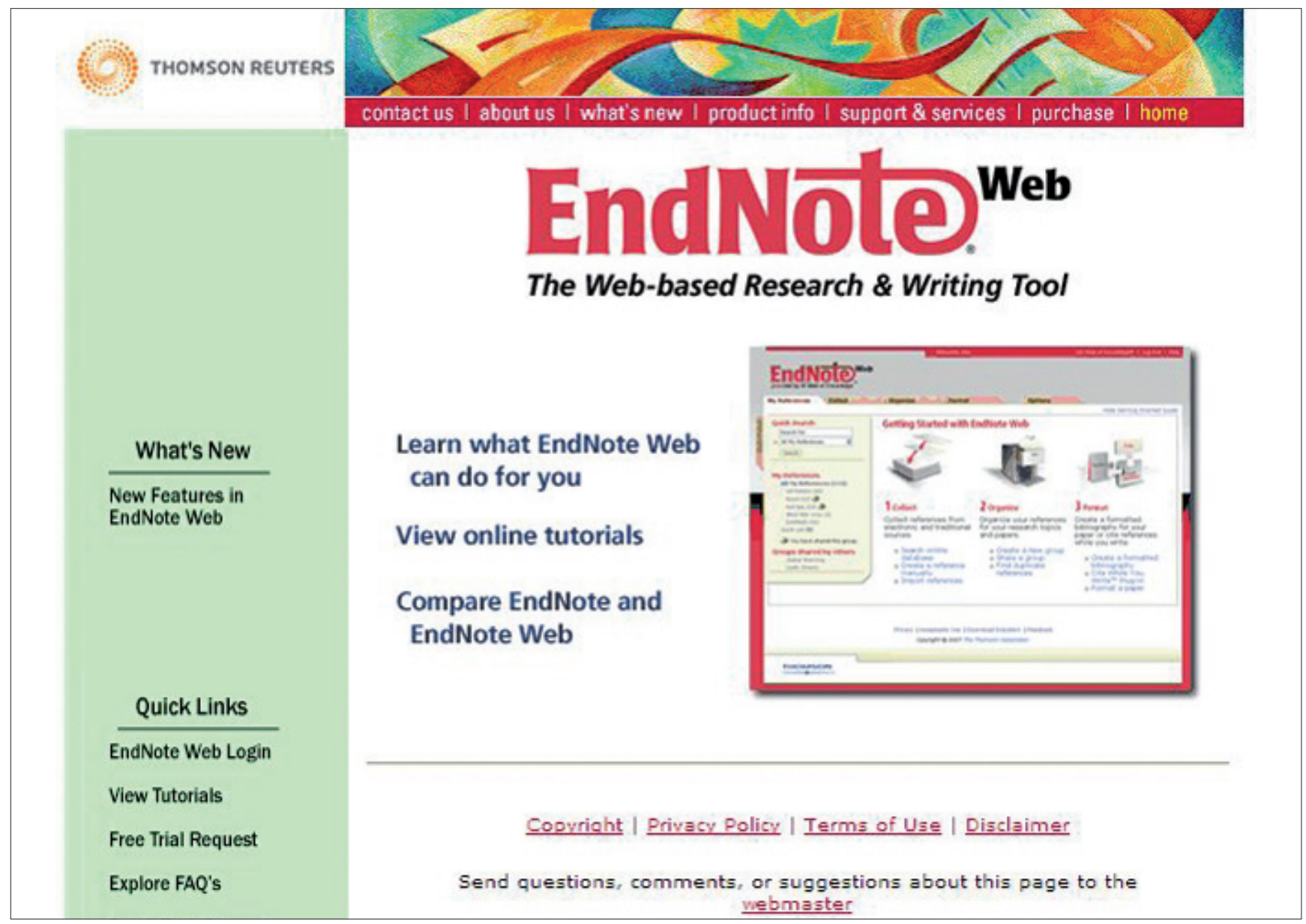

http://www.endnoteweb.com/

Los gestores más utilizados por su calidad, prestaciones y por las mejoras introducidas en los últimos años son RefWorks, EndNote web y Zotero. Poseen funciones similares aunque su nivel de prestaciones es diferente, por lo que es necesario evaluar sus aspectos más destacados si se quieren conocer sus posibilidades y su capacidad de adaptación a diferentes contextos de investigación. A continuación se exponen diversos parámetros de valoración aplicables a estos tres programas, pero extrapolables a otros de similares características.

\section{Evaluación}

\subsection{Características generales}

\section{Tipo de licencia}

RefWorks requiere suscripción mediante una licencia de pago anual, aunque también está disponible por un período de evaluación de 15 días. Una buena parte de las universidades españolas mantiene la suscripción institucional anual.

EndNote web es gratuito, pero con grandes limitaciones como la disponibilidad de un número máximo de registros (10.000), o un número limitado de carpetas a compartir (100).

Por último, Zotero es software libre con licencia abierta, lo que garantiza su completo uso y mejora por cualquier persona. Su limitación reside en que al ser una extensión de Mozilla FireFox sólo funciona desde este navegador.

Cada entidad deberá tomar en consideración los pros y los contras de estos programas para, en función de las necesidades de sus investigadores y su política de información, utilizar uno u otro.

\section{Lenguajes de trabajo}

Hasta hace apenas dos años los gestores no estaban disponibles en otra lengua que no fuera el inglés, incluso los propios manuales y guías de uso solamente estaban disponibles en esta lengua. Había alguna traducción no oficial, hecha por personas que habían trabajado con estas aplicaciones. RefWorks fue el primero en ofrecer una versión en castellano, y Zote$r o$ la tiene desde hace apenas un año. Esto ha favorecido el uso de gestores por parte de muchos investigadores, ya que a veces resulta complejo dominar la operatividad de un sistema cuando está en un idioma diferente al del usuario. Solamente EndNote no presenta versión en castellano, aunque tiene una comprensión fácil y bien estructurada por tareas.

\section{Versión web}

Parece lógico pensar que los gestores de última generación se integran en la Web como un elemento multiherrramienta, aunque hay que decir que su integración se realiza de formas diferentes. 


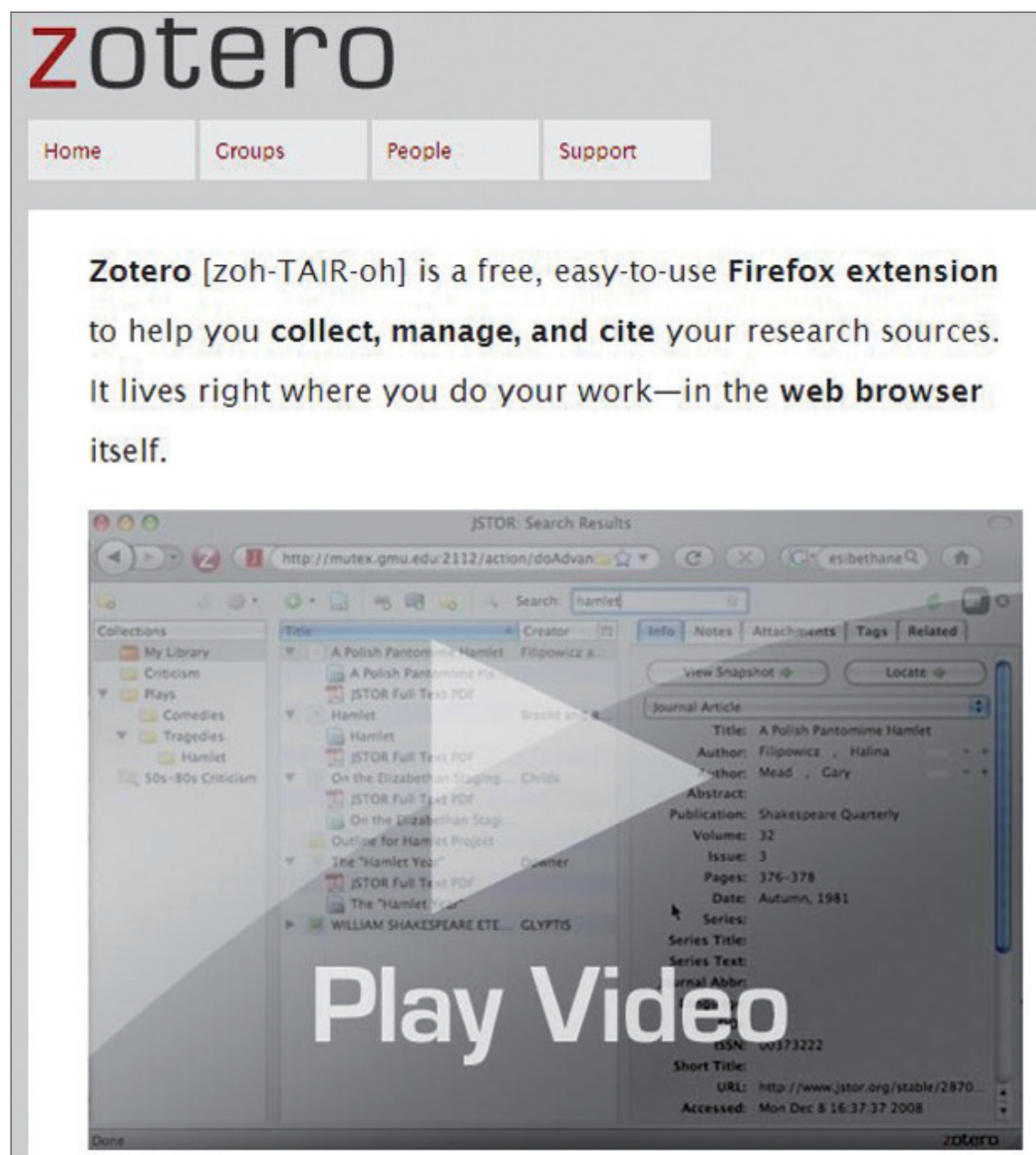

\section{Features}

Automatically capture citations

Remotely back up and sync your library

Store PDFs, images, and web pages

Cite from within Word and Openoffice

Take rich-text notes in any language

Wide variety of import/export options

Free, open source, and extensible

Collaborate with group libraries
Organize with collections and tags

Access your library from anywhere

Automatically grab metadata for PDFs

Use thousands of bibliographic styles

Instantly search your PDFs and notes

Advanced search and data mining tools

Interface available in over 30 languages

Recommendation engine and RSS feeds

\section{download 1.0}

Latest version: $1.0 .10-$ maximum stability

\section{try out 2.0 beta}

Latest version: $2.0 \mathrm{b5}$ - newest features

Which version should / use?

Having trouble installing?

\section{Minimum Requirements}

Firefox 3.0 for Windows, Mac, or Linux

\section{Zotero News}

Help Zotero by Donating to the Center For History and New Media June 3 , 2009

One Week Left To Apply For Summer Zotero Workshop May 26, 2009

Zotero 2.0 Mothership Lands May 14 2009

more zotero news..

\section{Produced by}
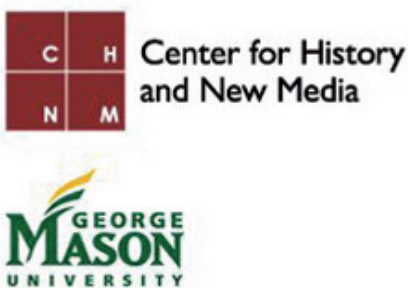

Sponsors

The

Andrew W.

Mellon

Foundation

http://www.zotero.org/

RefWorks y EndNote web lo hacen a través de una web a la que se accede mediante contraseña y password. Esto tiene ventajas, el que pueda estar siempre disponible desde diferentes terminales o lugares, e inconvenientes, como la limitación de no disponer en un momento determinado de una conexión a internet, cosa que impediría tener acceso a nuestra bibliografía, y que tengamos que depender de gestores con versión local. EndNote dispone de versión local, aunque mediante licencia adicional.

Zotero también se integra en la Web, pero de manera diferente, ya que se trata de un pequeño programa que instala una especie de barra de herramientas o extensión en la Web que, como dijimos anteriormente, sólo funciona con el navegador de Mozilla. A partir del lanzamiento de la versión 2, ya disponible en beta desde mayo de 2009, este gestor es capaz de sincronizar (actualizar) automáticamente las versiones locales en diferentes ordenadores con la versión web.

\section{Versión local}

Ya hemos señalado que solamente EndNote dispone de versión local: EndNoteX2. Ésta tiene una interfaz de comunicación bidirec- 
cional entre la versión local y la web. Zotero, al ser una extensión del navegador, comparte algunas de las particularidades, ventajas e inconvenientes de las versiones local y web, por ejemplo la imposibilidad de compartir datos a distancia, o la disposición de un sistema local que permite tener una copia independientemente de tener o no conexión. Además aporta una solución muy interesante, como es la realización de copias -que denomina instantáneas- de las páginas html, que podemos utilizar posteriormente aunque no tengamos acceso a ellas o conexión a la Red.

\section{"Los gestores bibliográficos actuales se orientan más a facilitar la tarea de los usuarios noveles"}

\section{Usabilidad}

Las capacidades de uso han mejorado mucho respecto a las anteriores versiones, ya que estos programas se orientan cada vez más a facilitar la tarea de usuarios no especialmente diestros en el manejo de sistemas de información. La Web ha permitido una evidente mejora en cuanto a la presentación física, organización y estructuración interna, y sobre todo a la manera, cada vez más intuitiva, de recopilar información de las fuentes digitales. Se ha pasado del manejo de cientos de filtros de información y conocimiento de los formatos de importación a la posibilidad de incorporar datos con un solo clic de ratón, y a la generalización de las salidas en un formato común comprensible por todos los gestores de referencias.

Esta sencillez ha ido en detrimento sin embargo de otras operaciones que resultaban complejas para un usuario no avanzado, pero que son muy necesarias para quienes gestionan fuentes de información o elaboran estudios bibliométricos, como son la imposibilidad de estos nuevos productos de crear formatos personalizados de entrada, salida y edición de información e importación. Muchos profesionales de la información utilizan gestores de última generación para incorporar datos, pero no para implementar servicios, por lo que siguen utilizando gestores clásicos como ProCite o EndNote como complemento de los de última generación.

Destaca la fácil incorporación de datos que proporciona Zotero. $\mathrm{La}$ idea de que aparezca un icono en la barra de direcciones que identifica el producto (libro, documento, película, carpeta) hace especialmente sencillo su manejo. Esto ha hecho posible que bases de datos (ISBN) o sitios comerciales (Amazon, YouTube) que no disponían de formatos de salida puedan ser recopilados automáticamente. Sin embargo el problema surge cuando no identifica una fuente.

Hay que destacar asimismo el buen diseño de EndNote web en cuanto a su estructura formal mediante pestañas que facilitan las tareas básicas del gestor: recogida de datos, organización y explotación (es decir, entrada, almacenamiento y salida). Se trata de una organización clara y unívoca. Otro aspecto muy diferente es el que presenta, por ejemplo, RefWorks donde aparece un menú desplegable que muestra una organización poco clara y a veces redundante, ya que las mismas funciones están en diferentes desplegables sin una razón justificada.

\section{Limitaciones}

Las limitaciones que imponen determinados productos hay que valorarlas en función de lo que queremos y lo que necesitan nuestros usuarios. Disponer de un producto gratuito -que no es sinónimo de software libre- como es el caso de EndNote web no siempre es la mejor solución, ya que es difícil evaluar si 10.000 registros es un número suficiente para que un investigador disponga de su bibliografía, y más cuando se estudia en campos cada vez más interdisciplinares y en distintos proyectos.

\subsection{Importación de datos}

\section{Recursos compatibles}

La compatibilidad de los gestores guarda una relación directa con los intereses comerciales de cada empresa distribuidora. Esto se traduce en que, por ejemplo, Thomson facilita la exportación directa desde ese proveedor de información a su gestor de referencias, en este caso EndNote; de la misma manera los productos informativos de ProQuest facilitan la exportación al gestor $R e$ fWorks de la misma multinacional. Para aquellos productos que no son distribuidos por estas empresas la única posibilidad es la de recurrir a la exportación de los recursos en RIS y a su importación posterior desde el gestor (ver tabla 1).

Vemos que de los productos analizados, RefWorks es directamente compatible con 7 de ellos, EndNote con 8, y Zotero con 15. En este sentido Zotero es por tanto el gestor que tiene mayor capacidad de interacción con las fuentes de información. Además destaca por su compatibilidad con fuentes comerciales que ningún otro gestor facilita.

\section{"Zotero tiene capacidad de interacción con casi todas las fuentes de información"}

\subsection{Control de autoridades}

La posibilidad de generar índices de los campos más relevantes 


\begin{tabular}{|l|l|l|l|}
\hline \multicolumn{1}{|c|}{ Fuente } & RefWorks & \multicolumn{1}{|c|}{ EndNote } & \multicolumn{1}{c|}{ Zotero } \\
\hline Amazon & No & No & Directa \\
\hline CSA & Directa & Indirecta & Directa \\
\hline Csic & Indirecta & Indirecta & Indirecta \\
\hline Dialnet & No & No & Directa \\
\hline Ebsco & Indirecta & Directa & Directa \\
\hline E-LIS & Indirecta & Indirecta & Directa \\
\hline Elsevier - Science direct & Directa & Indirecta & Directa \\
\hline Emerald & Indirecta & Indirecta & Directa \\
\hline Flickr & No & No & Directa \\
\hline Google Scholar & Directa & Directa & Directa \\
\hline IEEE & Directa & Directa & Directa \\
\hline J stor & Directa & Indirecta & Indirecta \\
\hline opacs & Directa - & Directa & Directa - (no \\
\hline OVID & Indirecta & todos) \\
\hline ProQuest & Directa & Directa & Directa \\
\hline Springer (Brill) & Directa & Directa & Indirecta \\
\hline Swets & Indirecta & Indirecta & Directa \\
\hline Web of Knowledge & Indirecta & Indirecta & Indirecta \\
\hline Wiley & Indirecta & Directa & Directa \\
\hline YouTube & Indirecta & Indirecta & Directa \\
\hline & No & No & Directa \\
\hline
\end{tabular}

Tabla 1

era una de las particularidades de los gestores de referencias clásicos. Éstos permitían la elaboración de estudios bibliométricos de la producción científica en un campo del saber o en una región determinada; esta peculiaridad se ha visto sacrificada en los gestores de nueva generación a favor de una simplificación en el uso por parte de investigadores que utilizan los gestores no para analizar datos, sino para disponer de su bibliografía personal.

El gestor que mejor responde a un sistema de control de autoridades es RefWorks, que genera índices hipertextuales de autores, descriptores y revistas, con el número de frecuencias de cada término, pudiéndose eliminar o editar cada término del índice. Lo que no podemos es generar salidas de listas de términos ordenadas por frecuencia de aparición. Los otros dos gestores -EndNote web y Zotero- ni siquiera generan índices de frecuencias, aunque al disponer de versión local el primero es quizá el que aporta mejores prestaciones para este tipo de cuestiones, ya que aparte de tener los índices básicos que genera automáticamente el sistema (autores, títulos, descriptores) permite generar índices de cada uno de los campos y exportarlos a una hoja de cálculo (Excel) para operar con ellos y generar gráficos. Zotero dispone de un sistema de etiquetado que denomina marcas pero que es muy básico, algo similar a las tags de los recursos web, con la posibilidad de añadir o modificar marcas.

\section{"RefWorks es el gestor que mejor responde a un sistema de control de autoridades"}

\subsection{Control de duplicados}

Otra cuestión destacada a tener en cuenta cuando recopilamos información de diferentes fuentes de información, que a veces tienen un alto nivel de solapamiento, es la posibilidad de detectar y descartar registros duplicados para evitar la redundancia de datos en el gestor. La única posibilidad que presenta Zotero para encontrar duplicados es desde la parte central donde se encuentran organizados los documentos. Ni RefWorks, ni EndNote web dan la posibilidad de descartar los duplicados a priori. En RefWorks podemos ir a la opción "ver" para buscar los duplicados exactos o casi exactos y con un solo clic eliminar todos los encontrados. La versión web de EndNote puede localizar los duplicados de una manera muy similar a como lo hace RefWorks. En cuanto al control de duplicados son más versátiles los gestores clásicos que pueden descartarlos durante la importación de datos.

\subsection{Citas}

Una de las funciones básicas de un gestor de referencias es facilitar la inclusión de citas bibliográficas en los formatos normalizados en los trabajos de investigación. Todos ellos ofrecen dos formas básicas: incluir citas en el propio texto, o bien generar una bibliografía para añadir al final del documento.

Para ello disponen de unas extensiones o programas que se integran en los propios procesadores de texto mediante una barra de herramientas con distintos iconos y que facilitan la tarea de citar un documento en el formato elegido por el usuario. Debemos destacar dos cuestiones: por una parte EndNote $w e b$ da la opción de importar los datos de una bibliografía preexistente, es decir, permite a partir de una bibliografía citada en un documento generar registros de estas referencias en la base de datos, aunque no lo hace con la eficiencia que sería deseada, ya que pocos documentos tienen una bibliografía citada con precisión. Y por otra Zotero, como corresponde a un programa de soft- 
ware libre, puede integrarse en el gestor de OpenOffice.

Todos ellos tienen cientos de estilos de citas, pero una cuestión interesante para el investigador en el ámbito europeo, es que sólo $R e$ fWorks y Zotero, en su última versión, son capaces de citar de manera automática en formato ISO. Una crítica frente a los gestores clásicos es que no podemos generar nuestros propios formatos de salida.

\subsection{Elementos Web 2.0}

Una característica de los nuevos tiempos es lo que se ha denominado Web social compartida o Web 2.0. Los nuevos gestores están cada vez más orientados a integrarse en esta línea de actuación.

\section{Área compartida}

Casi todos los gestores contemplan la posibilidad de compartir carpetas. Este es un elemento interesante cuando se trabaja con otros colegas del mismo departamento o bien de otros lugares geográficos ya que pueden disponer de una bibliografía común para ser utilizada por todos; el problema está en que los investigadores deberán utilizar el mismo producto, es decir si se trata de RefWorks (RefShare) tendrán que disponer todos ellos de una cuenta en el sistema. Según los permisos que el usuario de RefWorks conceda, los demás podrán ver, imprimir, exportar o generar listas de referencias desde RefShare. El área compartida de EndNote web presenta características muy similares a ésta, solo que al ser un programa gratuito cualquier usuario puede tener carpetas compartidas con otro que tenga cuenta en el programa. El método es muy simple, únicamente deberá incluir el correo electrónico con el que el otro usuario se haya inscrito. Zotero acaba de incorporar un área compartida con todas las posibilidades de las redes sociales, es decir hace posible a los usuarios compartir contenidos, o crear sus propios grupos de trabajo.

\section{Sindicación de contenidos}

El RSS se ha convertido en uno de los elementos clave para la difusión de la información en la Web 2.0. Todos los navegadores disponen de lectores de noticias con tecnología RSS que permiten que nos suscribamos a estos canales y dispongamos de información actualizada con un simple golpe de ratón: noticias, blogs, empleo, fuentes de información especializadas como revistas electrónicas. Casi todos los paquetes de revistas científicas tiene el famoso icono naranja para sindicar sus contenidos.

Si esto lo enmarcamos dentro del entorno de los gestores de referencias significa que si uno de ellos dispone de lector de canales RSS nos va a permitir importar las nuevas referencias sin necesidad siquiera de ir al recurso; por ejemplo, si nos suscribimos a la revista Journal of documentation, cuando ésta disponga de nuevos contenidos veremos junto al icono RSS el título en negrita y simplemente picando en él, importaremos las nuevas referencias de los artículos editados, así como el enlace al documento completo, más sencillo imposible.

De los gestores analizados de momento sólo uno -RefWor$k s$ - cuenta con lector RSS; además incorpora la posibilidad de hacer sindicación a partir de RefShare, de esta manera las personas que comparten carpetas saben en todo momento cuando se han incluido nuevas referencias; lo que le proporciona una ventaja competitiva importante respecto a los otros. No obstante Zotero ya ha anunciado la inminente incorporación de canales RSS en la versión 2.

\section{Posibilidad de trabajar sin co- nexión}

Los gestores de nueva generación con integración en la Web tienen notables ventajas, como el poder disponer del gestor sin tener que llevar el programa o la base de datos allá donde nos desplacemos, y tampoco tenemos que instalar ningún programa en nuestro ordenador; pero esta ventaja a veces puede suponer un contratiempo, si por las razones que sea no disponemos de acceso a internet. Esta cuestión la resuelven bien EndNote web que dispone de versión local, y Zotero, ya que al ser una extensión local dentro del navegador FireFox siempre lo tendremos disponible, incluso si no tenemos red. Otra cuestión interesante es que Zotero puede hacer imágenes de los documentos html, con lo cual aunque no tengamos red podremos ver el documento a texto completo si hemos generado previamente esa imagen. Otra posibilidad es que tanto $R e$ fWorks como Zotero permiten integrar documentos (word, pdf, ppt...) en las propios registros. Una funcionalidad muy útil e innovadora que ha incorporado la reciente versión de Zotero es la detección automática de metadatos pdf, es decir, si tenemos varios documentos en pdf en nuestro ordenador podemos arrastrarlos hasta el gestor, y este buscará los metadatos de cada documento en Google Scholar.

\section{Posibilidades de interacción con motores (Google Académico)}

La versión de Google para científicos, Google Scholar, nos da la posibilidad de exportar datos a gestores bibliográficos. Para ello hay que configurar en el área de preferencias la exportación directa a gestores, en concreto a EndNote, y RefWorks, de manera que cuando hacemos una búsqueda en este sistema nos aparece un hiperenlace en cada documento que nos dice "Importar a RefWorks". Zotero también reconoce los documentos de Google Académico mediante el sistema de icono de carpeta y tipos de documento, clásico de este gestor.

\section{Recursos comerciales}

Antes hemos mencionado que cada vez existe una diversificación mayor de las fuentes de informa- 
ción. Hasta hace bien poco los gestores sólo trabajan con fuentes de información del ámbito estrictamente científico. En la actualidad se abre la posibilidad de que otro tipo de fuentes de interés para el investigador como son presentaciones SlideShare, tutoriales en vídeo de YouTube o libros de reciente aparición en fuentes comerciales como Amazon, que aún no han sido recogidas por los servicios científicos de apoyo a la investigación puedan ser integradas por los gestores. En este sentido el gestor más avanzado es Zotero, que recoge de manera directa la información es esas fuentes.

\section{"Las organizaciones deben valorar la adecuación de cada gestor a las necesidades concretas de sus investigadores"}

\section{Capacidad para incluir sugeren- cias}

La Web 2.0 proporciona un entorno cooperativo en el que los usuarios colaboran para mejorar recursos y servicios, como ocurre en los sistemas denominados wiki. Esta posibilidad está definida especialmente en el ámbito del software libre y es el caso de Zotero, que forma parte de un sistema de código abierto que da a los usuarios más avanzados la posibilidad de ir optimizando el programa, de enviar a los administradores sugerencias para que incluyan nuevos productos y fuentes documentales que aún no son compatibles con Zotero, simplemente enviando un mensaje a la dirección de correo que nos indica. De esta manera ha sido posible la traducción del programa a una amplia variedad de idiomas (30 lenguas).

\section{Valoración general}

Después de un análisis comparativo de los diferentes gestores, sustentado en la experiencia que nos proporciona el trabajo diario como profesionales de la información, pasaremos a valorar, de manera general los diferentes productos analizados. Es difícil concretar cuál de ellos es el producto más completo, unos y otros tienen características relevantes de las que no dis- pone el resto, y viceversa. Hay que decir que hay aspectos destacados en cada uno de ellos. RefWorks tiene una particularidad básica que es la posibilidad de leer fuentes RSS; Zotero aventaja al resto en su capacidad para trabajar con fuentes de información del nuevo contexto tecnológico; y EndNote destaca, a nuestro parecer, por su buena organización y usabilidad; además de por disponer de una versión local que se complementa e integra con la versión web.

\section{"EndNote destaca por su buena organización y usabilidad, y por disponer de una versión local”}

Hay que señalar que casi todos ellos han sacrificado buena parte de las posibilidades de personalización que tenían los gestores clásicos en la generación de productos de entrada y salida de datos, para conseguir productos más fácilmente utilizables por un usuario medio sin grandes destrezas en el manejo de información, y que lo utiliza

\begin{tabular}{|c|c|c|c|c|c|c|c|c|c|c|c|}
\hline Gestor & Compañía & $\begin{array}{c}\text { Año de } \\
\text { apari- } \\
\text { ción }\end{array}$ & $\begin{array}{c}\text { Ultima } \\
\text { ver- } \\
\text { sión }\end{array}$ & Coste & Licencia & $\begin{array}{l}\text { Caracte- } \\
\text { rísticas }\end{array}$ & $\begin{array}{c}\text { Sistema } \\
\text { opera- } \\
\text { tivo }\end{array}$ & \begin{tabular}{|c|} 
Formatos \\
de ex- \\
portacion
\end{tabular} & $\begin{array}{c}\begin{array}{c}\text { Formatos } \\
\text { de im- } \\
\text { portacion }\end{array} \\
\end{array}$ & $\begin{array}{l}\text { Formatos } \\
\text { de salida }\end{array}$ & $\begin{array}{l}\text { Docs. } \\
\text { Salida }\end{array}$ \\
\hline EndNote & $\begin{array}{c}\text { Thomson } \\
\text { Corpora- } \\
\text { tion }\end{array}$ & 1988 & $x 2$ & $299 \$$ & $\begin{array}{c}\text { Propie- } \\
\text { taria }\end{array}$ & Local & $\begin{array}{l}\text { Windows } \\
\text { Mac OsX }\end{array}$ & \begin{tabular}{|c|} 
BibText, \\
EndNote/ \\
Ref Man, \\
Medline, \\
ris y otros
\end{tabular} & \begin{tabular}{|c|} 
CSA, ISI, \\
Medline, \\
Ovid, \\
PubMed, \\
ris, y \\
otros \\
\end{tabular} & $\begin{array}{c}\text { APA, } \\
\text { Chicago, } \\
\text { Harvard, } \\
\text { MLA y } \\
\text { otros }\end{array}$ & $\begin{array}{l}\text { html, rtf, } \\
\text { txt, xml }\end{array}$ \\
\hline $\begin{array}{l}\text { EndNote } \\
\text { web }\end{array}$ & \begin{tabular}{|c|} 
Thomson \\
Corpora- \\
tion
\end{tabular} & 2002 & 2.4 & $\begin{array}{l}\text { Gratis, } \\
\text { con } \\
\text { límites }\end{array}$ & Gratuito & $\begin{array}{l}\text { Versión } \\
\text { Web }\end{array}$ & \begin{tabular}{|c|} 
No \\
depende \\
de SO
\end{tabular} & \begin{tabular}{|c|} 
BibText, \\
EndNote/ \\
Ref Man, \\
Medline, \\
ris y otros
\end{tabular} & \begin{tabular}{|c|} 
CSA, ISI, \\
Medline, \\
Ovid, \\
PubMed, \\
RIS, y \\
otros
\end{tabular} & $\begin{array}{c}\text { APA, } \\
\text { Chicago, } \\
\text { Harvard, } \\
\text { MLA y } \\
\text { otros }\end{array}$ & $\begin{array}{c}\text { html, } \\
\text { html, rtf, } \\
\text { txt, xml }\end{array}$ \\
\hline RefWorks & $\begin{array}{c}\text { RefWorks } \\
\text { ProQuest } \\
\text { LLC }\end{array}$ & 2001 & 2008 & $\begin{array}{c}100 \$ \\
\text { año }\end{array}$ & $\begin{array}{c}\text { Propie- } \\
\text { taria }\end{array}$ & $\begin{array}{l}\text { Versión } \\
\text { Web }\end{array}$ & \begin{tabular}{|c|} 
No \\
depende \\
de SO
\end{tabular} & $\begin{array}{c}\text { BibTex, ris } \\
\text { y otros }\end{array}$ & $\begin{array}{c}\text { CSA, ISI, } \\
\text { Ovid, ris, } \\
\text { y otros }\end{array}$ & $\begin{array}{c}\text { ISO, APA, } \\
\text { Chicago, } \\
\text { Harvard, } \\
\text { MLA y } \\
\text { otros }\end{array}$ & $\begin{array}{c}\text { html, } \\
\text { html, rtf, } \\
\text { txt, xml, } \\
\text { rss }\end{array}$ \\
\hline Zotero & $\begin{array}{l}\text { George } \\
\text { Mason } \\
\text { Univer- } \\
\text { sity }\end{array}$ & 2006 & $\begin{array}{c}2.0 b 4 \\
\text { may } \\
2009\end{array}$ & Gratis & $\begin{array}{c}\text { Software } \\
\text { libre }\end{array}$ & $\begin{array}{c}\text { Extensión } \\
\text { FireFox }\end{array}$ & $\begin{array}{c}\text { Windows } \\
\text { Mac } \\
\text { Unix } \\
\text { Linux }\end{array}$ & \begin{tabular}{|c|} 
BibText, \\
EndNote/ \\
Ref Man, \\
ris, rdf y \\
otros
\end{tabular} & \begin{tabular}{|c|} 
ISI, Ovid, \\
PubMed, \\
ris, Marc, \\
rdf, Arxiv, \\
CiteSeer \\
\end{tabular} & $\begin{array}{c}\text { APA, } \\
\text { Chicago, } \\
\text { Harvard, } \\
\text { MLA y } \\
\text { otros }\end{array}$ & html, rtf \\
\hline
\end{tabular}

Tabla 2. Evaluación de gestores. Aspectos generales 


\begin{tabular}{|c|c|c|c|c|c|c|c|c|c|c|}
\hline Gestor & $\begin{array}{l}\text { Integración } \\
\text { procesador }\end{array}$ & $\begin{array}{l}\text { Versio- } \\
\text { nes }\end{array}$ & Lenguas & $\begin{array}{l}\text { Usabi- } \\
\text { lidad }\end{array}$ & $\begin{array}{l}\text { Limita- } \\
\text { ciones }\end{array}$ & $\begin{array}{c}\text { Recursos } \\
\text { compati- } \\
\text { bles } \\
\text { Direct. }\end{array}$ & $\begin{array}{c}\text { Bús- } \\
\text { queda } \\
\text { directa }\end{array}$ & $\begin{array}{l}\text { Control } \\
\text { de auto- } \\
\text { ridades }\end{array}$ & Citas & Web 2.0 \\
\hline EndNote & Word & $\begin{array}{l}\text { Local y } \\
\text { web }\end{array}$ & Inglés & Media & Ninguna & ISI & Sí & \begin{tabular}{|l|} 
Cambios \\
masivos \\
Crea \\
índices \\
Control \\
de dupli- \\
cados \\
\end{tabular} & $\begin{array}{l}\text { Citas al } \\
\text { final } \\
\text { Biblio- } \\
\text { grafía }\end{array}$ & $\begin{array}{l}\text { No compar- } \\
\text { tido } \\
\text {-Hipertexto }\end{array}$ \\
\hline $\begin{array}{l}\text { EndNote } \\
\text { web }\end{array}$ & Word & $\begin{array}{l}\text { Local y } \\
\text { web }\end{array}$ & Inglés & Buena & $\begin{array}{l}10.000 \\
\text { registros } \\
100 \text { cola- } \\
\text { boradores }\end{array}$ & ISI & Sí & \begin{tabular}{|l|} 
Cambios \\
masivos \\
Crea \\
índices \\
Control \\
de dupli- \\
cados \\
\end{tabular} & $\begin{array}{l}\text { Citas al } \\
\text { final } \\
\text { Biblio- } \\
\text { grafía }\end{array}$ & $\begin{array}{l}\text { Compartir } \\
\text { carpetas } \\
\text { - Hipertexto }\end{array}$ \\
\hline RefWorks & Word & Sólo web & Múltiples & Buena & Ninguna & $\begin{array}{l}\text { ProQuest, } \\
\text { CSA. Ovid, } \\
\text { Scopus, } \\
\text { Science } \\
\text { Direct, } \\
\text { Google } \\
\text { Académico }\end{array}$ & Sí & \begin{tabular}{|l|} 
Cambios \\
masivos \\
Crea \\
índices \\
Control \\
de dupli- \\
cados \\
\end{tabular} & $\begin{array}{l}\text { Citas al } \\
\text { final } \\
\text { Biblio- } \\
\text { grafía }\end{array}$ & $\begin{array}{l}\text {-Sindicación } \\
\text { de contenidos } \\
\text { - Compartido } \\
\text { - Importa } \\
\text { sitios web } \\
\text { - Hipertexto }\end{array}$ \\
\hline Zotero & $\begin{array}{l}\text { Word/ } \\
\text { OpenOffice }\end{array}$ & $\begin{array}{l}\text { Sólo web } \\
\text { / sincro- } \\
\text { nizada } \\
\text { versión } \\
\text { local }\end{array}$ & Múltiples & $\begin{array}{l}\text { Muy } \\
\text { buena }\end{array}$ & Ninguna & \begin{tabular}{|l|} 
Solicitud, \\
Recursos \\
comerciales \\
(Youtube, \\
Amazon, \\
Wikipedia, \\
Google \\
Scholar)
\end{tabular} & No & $\begin{array}{l}\text { Cambios } \\
\text { masivos }\end{array}$ & $\begin{array}{l}\text { Citas al } \\
\text { final } \\
\text { Biblio- } \\
\text { grafía }\end{array}$ & $\begin{array}{l}\text {-Trabaja sin } \\
\text { conexión } \\
\text { - Recursos } \\
\text { comerciales } \\
\text {-I Incluye no- } \\
\text { tas e instan- } \\
\text { táneas } \\
\text {-I mporta } \\
\text { sitios web } \\
\text { - Posibilidad } \\
\text { de introducir } \\
\text { sugerencias } \\
\text {-I ntegración } \\
\text { WordPress } \\
\text { - Red social }\end{array}$ \\
\hline
\end{tabular}

Tabla 3. Evaluación de gestores. Aspectos específicos

\begin{tabular}{|c|c|c|}
\hline Gestor & Puntos fuertes & Aspectos mejorables \\
\hline EndNote & $\begin{array}{l}\text { - Versión local y web } \\
\text { - Muy bueno para estudios bibliométricos (listas, } \\
\text { tablas por frecuencia) }\end{array}$ & $\begin{array}{l}\text { - Uso relativamente complejo } \\
\text { - Búsqueda compleja y poco eficaz }\end{array}$ \\
\hline EndNote web & $\begin{array}{l}\text { - Versión local y web } \\
\text { - Buena organización interna (pestañas, colores) } \\
\text { - Visualizar de dónde fue exportado cada registro } \\
\text { - Importar bibliografías de Word }\end{array}$ & $\begin{array}{l}\text { - Limitaciones en el número de registros } \\
\text { - Limitaciones en los usuarios a compartir (100) } \\
\text { - Limitaciones a } 25 \text { favoritos } \\
\text { - Compatibilidad por limitaciones comerciales } \\
\text { - Posibilidad de crear formatos propios de entra- } \\
\text { da y salida }\end{array}$ \\
\hline RefWorks & $\begin{array}{l}\text { - Sindicación de contenidos } \\
\text { - Formato ISO }\end{array}$ & $\begin{array}{l}\text { - No dispone de versión local } \\
\text { - Compatibilidad por limitaciones comerciales } \\
\text { - Complejidad importación indirecta } \\
\text { - Posibilidad de crear formatos propios de entra- } \\
\text { da y salida }\end{array}$ \\
\hline Zotero & $\begin{array}{l}\text { - Sin limitaciones comerciales } \\
\text { - Poder sugerir sitios } \\
\text { - Integra recursos comerciales } \\
\text { - Hacer instantáneas } \\
\text { - Usabilidad } \\
\text { - Posibilidad de introducir sugerencias } \\
\text { - Buen servicio soporte (tutoriales) } \\
\text { - Facilidad de exportación (barra de direcciones) } \\
\text { - Iconos tipo documento } \\
\text { - Detección automática metadatos }\end{array}$ & $\begin{array}{l}\text { - Control de autoridades } \\
\text { - Recursos aún no compatibles con Zotero } \\
\text { - Sólo compatible con FireFox } \\
\text { - Posibilidad de crear formatos propios de entra- } \\
\text { da y salida } \\
\text { - Mejorar el sistema de búsqueda }\end{array}$ \\
\hline
\end{tabular}


para disponer de su bibliografía y poderla citar adecuadamente en sus documentos de investigación.

Muchos profesionales de la información que trabajan en el ámbito de la documentación profesional utilizan estos gestores por su capacidad para recopilar datos, pero a la hora de generar productos documentales siguen transfiriendo estos datos a los sistemas clásicos como ProCite o EndNote X2.

De cualquier manera las instituciones u otras organizaciones deberán valorar las distintas características y prestaciones de estas herramientas y dar prioridad a aspectos relativos a licencias, y a la adecuación del producto a las necesidades concretas de sus investigadores.

\section{Notas}

1. RIS = Research Information Systems, Inc., siglas de la empresa que creó este formato

2. Manuales de estilo

ACS (American Chemical Society)

Handbook for authors of papers in the journals of the American Chemical Society. Washington, DC: ACS Publications, 1978, 119 pp.

AIAA (American Institute of Aeronautics and As tronautics)

http://www.aiaa.org/documents/publications/ journalstemp.dot

ALA (American Library Association)

http://www.ala.org/ala/mgrps/divs/rusa/resources/guidelines/index.cfm

ANSI (American National Standards Institute) American national standard for bibliographic references. New York: American National Standard Institute, 1977, 92 pp.

Chicago University Press manual of style http://www.chicagomanualofstyle.org/home.html

MLA (Modern Language Association) http://www.kent.ac.uk/uelt/ai/MLAstyle.pdf

UMI (University Films International)

Usado para tesis doctorales

Vancouver (Uniform requirements for medical journals)

http://www.ncbi.nlm.nih.gov/books/ bv.fcgi? rid=citmed.TOC\&depth $=2$

\section{Bibliografía}

Alonso-Arévalo, Julio. "ProCite 5.0: Guía". Biblioteca virtual en ciencias de la salud. Habilidades informacionales: recursos, metodología y técnicas de trabajo científico, 2006. http://eprints.rclis.org/archive/00007214/01/ProCite50.pdf

Alonso-Arévalo, Julio. "ProCite: Sistemas gestores de referencias bibliográficas". Biblioteca virtual en ciencias de la salud. Habilidades informacionales: recursos, metodología y técnicas de trabajo científico, 2006.

http://sabus.usal.es/site\%20med/descargas/ProCite\%202006_julio.pdf

Alonso-Arévalo, Julio. "RefWorks". Biblioteca virtual en ciencias de la salud. Información sanitaria en la Red. Nuevos recursos. Nuevos servicios, 2007.

http://sabus.usal.es/bib_virtual/doc/julio_gestores.zip

Alonso-Arévalo, Julio; López-Lucas, Jesús. "Gestores de referencias: Endnote, ProCite, RefWorks". Biblioteca virtual en ciencias de la salud. Información sanitaria en la Red. Nuevos Recursos. Nuevos Servicios, 2007.

http://sabus.usal.es/bib_virtual/doc/julio_gestores.zip

Alonso-Arévalo, Julio; López-Lucas, Jesús. "Selección, producción y edición de datos: los gestores de referencias bibliográficas: EndNote, ProCite, Reference Manager, BiblioScape/BiblioExpress. Normas de citación". Biblioteca virtual en ciencias de la salud. Habilidades informacionales: recursos, metodología y técnicas de trabajo científico, 2006.

http://sabus.usal.es/site\%20med/descargas/Manual_ProCite50_Julio\%20Alonso.pdf

Arencibia-Jorge, Ricardo; Araújo-Ruiz, JuanAntonio; Torricella-Morales, Raul-Gonzalo. "La nanotecnología como disciplina científica: un estudio bibliométrico del Web of Science en el período 1987-2004". Acimed, 2005, v. 13, n. 4. http://bvs.sld.cu/revistas/aci/vol13_4_05/ aci04405.htm

Arencibia-Jorge, Ricardo; Perezleo Solorzano, Ligeya; Araújo-Ruiz, Juan-Antonio. "Experiencias preliminares del Centro Nacional de Investigaciones Científicas en el uso de ProCite para la implementación de servicios de alto valor agregado". Acimed, 2003, v. 11, n. 6.

http://bvs.sld.cu/revistas/aci/vol11_6_03/ aci14603. htm

Armenteros-Vera, Ileana; Alfonso-Sánchez, Ileana. "Los gestores personales de bases de datos bibliográficas: conoce usted qué es y cómo se maneja el ProCite". Acimed, 2004, v. 12, n. 2.

http://bvs.sld.cu/revistas/aci/vol12_2_04/ aci06204..htm

Armenteros-Vera, Ileana; Alfonso-Sánchez, Ileana. "Los gestores personales de bases de datos bibliográficas". Wikilearning, 2006.

http://www.wikilearning.com/los_gestores_personales_de_bases_de_datos_bibliograficaswkc-7787.htm

Bravo-Toledo, Rafael. "Gestores personales de bases de datos bibliográficas". El profesional de la información, 1996, n. 10.

http://www.elprofesionaldelainformacion.com/ contenidos/1996/octubre/gestores_personales_ de_bases_de_datos_bibliogrficas.html
Codina, Lluís. "Reference Manager: herramientas para el trabajo intelectual". El profesional de la información, 2000, v. 9, n. 10, octubre, pp. 20-21.

Codina, Lluís. "Reference Manager: un sistema experto en procesamiento de bibliografías". El profesional de la información, 2002, v. 11, n. 3, mayo-junio, pp. 209-212.

Duarte-García, Emilio. "Gestores personales de bases de datos de referencias bibliográficas: características y estudio comparativo". El profesional de la información, 2007, v. 16, n. 6, pp. 647-656.

East, John W. "Z39.50 and personal bibliographic software". Library hi tech, 2003, v. 21, n. 1.

http://www.emeraldinsight.com/0737-8831.htm

Kessler, Jane; Van Ullen, Mary K. "Citation generators: generating bibliographies for the next generation". Journal of academic librarianship, 2005, v. 31, n. 4

http://www.sciencedirect.com/science/journal/00991333

López-Lucas, Jesús. "Gestores de referencias bibliográficas: utilidades de EndNote" Biblioteca virtual en ciencias de la salud. Información sanitaria en la Red. Nuevos Recursos. Nuevos Servicios, 2007.

http://sabus.usal.es/bib_virtual/doc/jesus_util_ end_note.pdf

Russo-Gallo, Patricia; Rodríguez-Gairín, Josep-Manuel; Sulé-Duesa, Andreu. "Laboratorio virtual de software libre para bibliotecas". El profesional de la información, 2008, v. 17, n. 1, pp. 71-77.

Serrat-Brustenga, Marta; Sunyer-Lázaro, Silvia. "Experiències en l'ús dels gestors de referències bibliogràfiques a la Biblioteca del Campus de Terrassa (BCT) de la UPC'. BiD: Biblioteconomia i Documentació, 2005, v. 14. http://www.ub.es/bid/14serrat.htm

Sociedad Valenciana de Patología Digestiva. Los gestores de referencias bibliográficas, 2007.

Torricella-Morales, Raúl-Gonzalo. "Aplicación de la mapificación conceptual en la creación de bases de datos temáticas especializadas: estudio de caso". Info: Congreso intl de información, 2004 , v. 5 .

http://www.congreso-info.cu/UserFiles/File/ Info/Info2004/Ponencias/106.pdf

Universidad de Sevilla. ProCite: Gestión de referencias bibliográficas. Universidad de Sevilla, 2006.

http://bib.us.es/aprendizaje_investigacion/publicar_citar/herramientas/common/procite.pdf
José A. Cordón-García, Helena Martín-Rodero, Julio Alonso-Aré- valo, Universidad de Salamanca. jcordon@usal.es helena@usal.es
alar@usal.es 